\title{
Determination of optimal placements of markers on the thigh during walking and landing
}

\author{
A. Thouzé 1-2-3 a, T. Monnet $^{1}$, M. Begon ${ }^{2-3}$, and M.T.G. Pain ${ }^{4}$ \\ ${ }^{1}$ Institut P' UPR 3346, Université de Poitiers, Bd M\&P Curie 86962 Futuroscope, France \\ ${ }^{2}$ Department of Kinesiology Université de Montréal C.P. 6128, Montreal H3C3J7, Canada \\ ${ }^{3}$ Centre de Recherche de l'Hôpital Sainte-Justine Montréal H3T 1C5, Canada \\ ${ }^{4}$ SSES, Loughborough University, Leics LE11 3TU, UK
}

\begin{abstract}
Kinematics of skin markers are affected by skin tissue artefact with respect to the bone during sports activities or locomotion. The purpose of this study is to determine the less disturbed marker's location for walking and landing. Twenty-six markers were put on the thigh of nine male subjects. Each subject performed a static trial, a setup movement for determining a functional hip joint centre and five walking and landing trials. The marker displacements were obtained by comparing recorded marker positions and solidified marker positions based on the geometry of the static acquisition. The markers were subsequently ranked from the worst to the least deformed. The ranking of each trial for each subject was analyzed with the concordance coefficient of Kendall and descriptive statistics were used to determine the most and the least disturbed markers. The results show reproducibility between trials for each subject for the two movements. Statistical analysis shows that the most deformed markers during walking were located close to the hip and knee joints whereas the least disturbed were on the mid-thigh. The landing analysis does not permit to determine the best markers from the worst.
\end{abstract}

\section{Introduction}

The measurement of three-dimensional kinematics of human locomotion using a motion analysis system requires skin markers put over the body segments. Markers are directly attached to the skin around the bony reliefs and are used with joint centres to build local systems of coordinates. These systems of coordinates are used to calculate the kinematics of body segments according to the functional anatomy (e.g. flexion-extension, abduction-adduction, and internal-external rotation). The main drawback is that skin markers are highly affected by soft tissue artefact which represent the major source of inaccuracy in human movement analysis [1]. The measured marker kinematics are not consistent with the assumption of a rigid body segment.

Methods for avoiding or reducing skin movement artefact exist. Pins may be screwed into the bone to obtain the actual bone kinematics; this approach is highly invasive. The intracortical pins and the required anesthesia cause discomfort that may affect gait pattern [2]. Bone kinematics can also be measured from medical imaging such X-ray and fluoroscopy but such analysis focuses on a single joint [3]. Based on mathematical and non-invasive approaches, kinematics of a cluster of markers (markers associated to one segment) can be solidified to consider the segment as a rigid body and improve the accuracy [4]. In addition, a minimum of four markers for each segment with an optimization procedure for calculating the roto-translation matrix reduces random and systematic

${ }^{a}$ arsene.thouze@gmail.com

This is an Open Access article distributed under the terms of the Creative Commons Attribution-Noncommercial License 3.0, which permits unrestricted use, distribution, and reproduction in any noncommercial medium, provided the original work is properly cited. 
error [5].Although soft tissue artefact are dependent on the marker locations, only a few papers have focused on optimal marker locations [6].

For gait analysis, the markers are usually put on femoral condyles and greater trochanter to define the thigh system of coordinates. However these locations have shown large displacements with respect to the bone: up to $30 \mathrm{~mm}$ for the marker located on the greater trochanter [1] which leads to an error of $5.8^{\circ}$ in knee flexion and up to $10 \mathrm{Nm}$ in knee moments. Since walking is a widely studied human movement, better marker location could improve the accuracy of analysis, especially in a clinical context. The purpose of this study is to assess the marker displacement due to soft tissue on the thigh during walking. Displacement is defined as the average Euclidean distance between an actual and a recall location from a rigid segment assumption. A second activity, landing, was also studied to analyze if soft tissue artifact are movement dependent. Twenty-six marker locations will be ranked for walking and landing to determine the worst and least affected area markers by soft tissue artefact.

\section{Methods}

A set of 26 markers was placed methodically - five lines of four to six equidistant markers (Fig. 1) on the thigh of nine male subjects. Each subject performed a static trial, five trials of walking at a self selected speed, and five landing trials from a $0.92 \mathrm{~m}$ high box. Kinematic data was collected at $300 \mathrm{~Hz}$ using a VICON motion analysis system of ten T40 cameras.
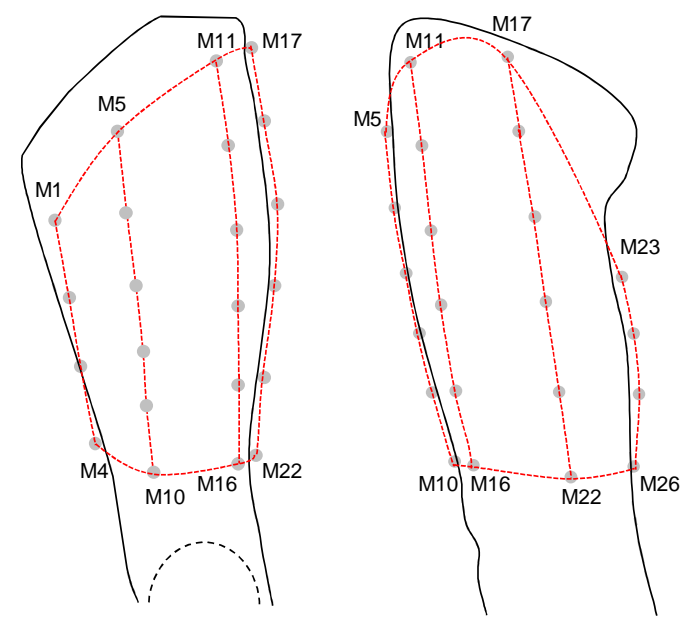

Fig. 1 Marker placement on the thigh

During the static trial in anatomical posture, the reference geometry of the 26 markers was acquired. From this record the local coordinate $\left({ }^{L} m_{i}\right)$ of each marker were calculated from its global coordinates $\left({ }^{G} m_{i}\right)$. The local frame $R_{L}$ of the thigh was created with all the markers [7]. The origin ${ }^{G} t$ of the local frame was determined as the centroid of the 26 markers and ${ }^{G} R_{L}$ is the rotation matrix from global frame:

$$
\begin{gathered}
{ }^{L} m_{i}={ }^{G} R_{L}\left({ }^{G} m_{i}-{ }^{G} t\right), \\
\text { with }{ }^{L} m_{i}=\left[\begin{array}{c}
{ }^{m} x_{i} \\
{ }^{m} y_{i} \\
{ }^{m} z_{i}
\end{array}\right]_{R_{L}},{ }^{G} m_{i}=\left[\begin{array}{c}
{ }^{m} x_{i} \\
{ }^{m} y_{i} \\
{ }^{m} z_{i}
\end{array}\right]_{R_{G}} \text {, and }{ }^{G} t=\frac{1}{n} \sum_{i=1}^{n}{ }^{G} m_{i}
\end{gathered}
$$


The displacement of each marker was calculated as the Euclidean distance between the actual $\left({ }^{G} m_{i}\right)$ and recalled $\left({ }^{G} r_{i}\right)$ positions (Eq. 2).

$$
d_{i}=\sqrt{\left({ }^{m} x_{i}-{ }^{r} x_{i}\right)^{2}+\left({ }^{m} y_{i}-{ }^{r} y_{i}\right)^{2}+\left({ }^{m} z_{i}-{ }^{r} z_{i}\right)^{2}},
$$

The average distance $(\bar{d})$ over a cycle defines the error associated with each marker. The markers were then ranked from the most disturbed to the least one. The displacement calculated in this paper does not correspond to soft tissue artefact since the reference was the global kinematics of all the markers in a static condition and not the bone kinematics.

The agreement of the marker ranking between the five walking trials of each subject was then assessed using Kendall's coefficient of concordance $(W)$. To test for statistical significance, $\chi^{2}$ is obtained from $W$ using the formula (Eq. 3):

$$
\chi^{2}=W \times k \times(N-1)
$$

where $k$ is the number of observations ( 5 trials) and $N$ is the number of markers. The critical value of the $\chi^{2}$ distribution was predetermined at 52.62 for $\alpha=0.01$ with 25 degrees of freedom. If the value of $\chi^{2}$ equals or exceeds the critical value, the marker order is not random. Then descriptive statistical - average plus or minus a standard deviation - analysis of the rankings will be used to determine the worst and the least deformed markers after checking that the each marker ranking follow a symmetrical distribution. The symmetry of a distribution is assessed using the skewness coefficient $\alpha_{3}$ which is determined by Eq 4:

$$
\alpha_{3}=\frac{m_{3}}{s_{x}{ }^{3}},
$$

where $m_{3}$ is the third order cumulant and $s_{x}$ is the variance. When $\alpha_{3} \rightarrow 0$, the distribution is considered as symmetric. The third order cumulant is calculated using Eq 5:

$$
m_{3}=\frac{1}{n-1} \times \sum_{i=1}^{n}\left(x_{i}-\bar{x}\right),
$$

where $n$ is the number of subjects, $x_{i}$ is the marker's ranking and $\bar{x}$ is this mean ranking.

Usually four to six markers are required to accurately calculate the roto-translation [8]. We tried to determine these markers for gait and landing for which the confidence interval did not overlap with that of the worst markers. The confidence interval was defined as $95 \%$ of the ranking distribution ( \pm 2 standard deviations).

\section{Results}

During walking (landing), the average marker displacement ranged from 3 to $25 \mathrm{~mm}$ (3 to $30 \mathrm{~mm}$ ). For ranking, the minimum value of $W$ for the intra-subject analysis was equal to 0.66 for a $\chi^{2}$ of 81.9 for walking and a $W$ of 0.89 for a $\chi^{2}$ of 108.5 for landing. As soon as the critical value of 52.62, for each subject the ranking is reproducible during the walking and landing trials.

Since the maximum value of $\alpha 3$ for was 1.4 rankings follow a symmetrical distribution, the worst and best marker locations could be determined using the confidence intervals (Fig 2a). The markers M11, M17, M5, and M10 are the most disturbed markers. These markers are located near the hip and 
knee joints (Fig. 2b) whereas the least disturbed markers are located on the middle anterior, lateral and posterior sides of the thigh (M3, M21, M8, M7, and 25).
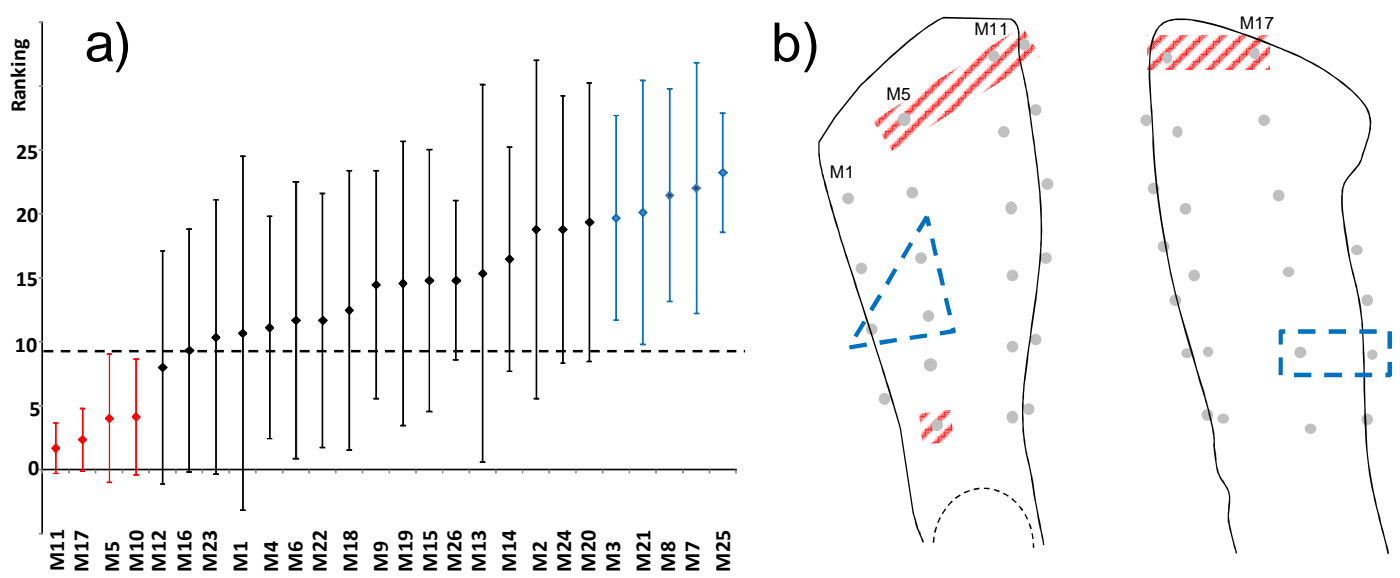

Fig. 2 a) Average ( \pm 2 SD) marker ranking for walking. b) Frontal and lateral views of the thigh with the least disturbed marker area (blue dashed rectangles) and the most disturbed marker area (red striped rectangles) during walking.

For landing the maximum value of $\alpha_{3}$ was 0.05 . Then rankings follow a symmetrical distribution, the worst marker locations could be determined using the average and the confidence intervals (Fig. 3). Since the inter-subject variability was higher than during walking, it was impossible to determine four to six markers that showed better ranking than other locations.

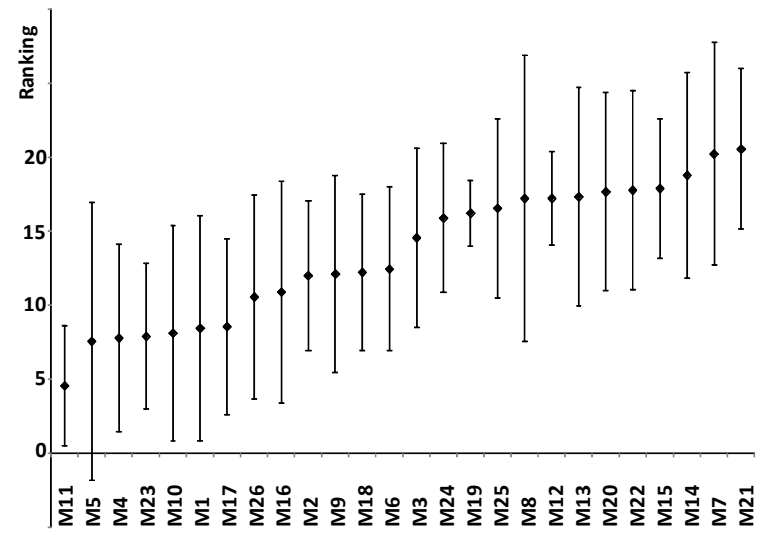

Fig. 3 Average ( \pm 2 SD) marker ranking for landing.

\section{Discussion}

This study aimed at finding markers that could be used to accurately estimate rigid body thigh kinematics during walking and landing. The statistical analysis has shown that all marker rankings between the trials of each subject were consistent in both movements. For walking analysis, the most rigid marker corresponds to the mid-thigh: front, external, and back sides. However in landing, the analysis did not highlight any accurate marker set. 
The maximal deviations ( $25 \mathrm{~mm}$ for walking vs $30 \mathrm{~mm}$ for landing) were located close to the hip and knee areas. The displacement obtained does not quantify soft tissue artefact but corresponds to the difference between reference kinematics derived from all markers and individual marker motion. The displacement magnitudes measured here correspond well with those obtained in the literature regarding the quantification of soft tissue artefact [9]. Soft tissue artefact using external fixators or intracortical pins were up to $20 \mathrm{~mm}$ [10]. Our results are also consistent with those of Reinschmidt et al. [2]. Using intracortical pins, they have highlighted that during gait large skin movement artefacts are associated with the markers placed at the greater trochanter or close to the femoral condyles.

In general, the results of this study suggest using marker locations M3, M21, M8, M7, and M25 for walking analysis which defines the areas shown in Fig. 2a. These marker positions are the inputs for the process of joint kinematic calculations. Several intermediate steps, i.e. estimation of the joint centre locations using a functional method and calculation of the rotation matrices, depend on marker coordinates and affect joint kinematics. For describing the thigh kinematics, markers are commonly located on the greater trochanter (M11 and M17) and on the femoral condyles (M10 and M16) [11] but this study highlights that these locations are highly affected by skin movement artefacts. Instead of using these markers for gait analysis, the position and orientation of the thigh should be determined from markers placed on the mid-thigh. To define a thigh system of coordinates that respects the functional anatomy, the greater trochanter and condyles can be located during the static trial using the Calibrated Anatomical System Technique method [12]. This method is recommended for locating bony landmarks in the system of coordinates built with the markers put all over the segment. During landing the wide range of flexion angles and peak ground reaction forces were not controlled, subject may have performed different types of landing. Due to these differences in kinematics and magnitude of contact forces, the skin marker deformation is more reproducible for walking than landing. That showed the movement specificity of soft tissue artefact.

At least three markers are needed to calculate the rotation matrix but it was shown that increasing the number of markers improves accuracy [5]. Moreover, the precision of the hip joint centre location using a functional method is increased by using more than three markers [8]. The results of this study could help in determining the best locations for these extra-markers. Although the results presented in this paper are consistent for a walking analysis, they should not be extrapolated to other movements because soft tissue artefact is movement specific [7]. This method cannot be proposed to explicitly find the most useful marker set to procure true femoral kinematics. It is a limitation of this study because no gold standard was used to obtain the femur kinematics and compare with our results. However, since the approach presented here is not invasive it can be applied in numerous situations where intracortical pins or fluoroscopy is not feasible.

Additional acquisitions with various somatotypes, several participants, and several movements are needed to extend these results and make further general recommendations. For a larger heterogeneous population, the marker deviations for each subject could be expressed in percentage terms with respect to the largest deviation. Hence, parametric statistics could be applied to these normalized values to highlight differences between the locations. The knowledge of soft tissue artefact is important to not only assess the accurate kinematics of the skeleton but for other applications, especially for inverse dynamics, the wobbling masses motion needs to be considered as well [13]. Further papers on this topic will try to assess both soft tissue artefact and wobbling mass motions. 


\section{Conclusion}

A method based on marker rankings to determine the worst and least disturbed markers during gait analysis was proposed in this study. For all subjects, marker ranking were similar between trials. The descriptive statistical analysis highlights that the best marker locations for gait analysis are on the mid-thigh (front, external, and back sides). As shown in previous studies, the worst markers were located near the hip and knee joint centres.

\section{References}

1. A. Cappozzo, F. Catani, A. Leardini, M.G. Benedeti, U. Della Croce, Clinical Biomechanics, 11, (1996)

2. C. Reinschmidt, A. Bogert, A. Lundberg, B. Nigg, N. Murphy, A. Stacoff, A. Stano, Gait \& Posture, 6, (1997)

3. E.H. Garling, B.L. Kapteina,, B. Mertensc, W. Barendregtd, H.E.J. Veeger, R.G.H.H. Nelissena, E.R. Valstara, Journal of Biomechanics, 40, (2007)

4. L. Chèze, B.J., Fregly, J., Dimnet, Journal of Biomechanics, 28 (1995)

5. J.H., Challis, Journal of Biomechanics, 28, (1995)

6. V. Camomilla, M. Donati, R. Stagni, A Cappozzo, Journal. of Biomechanics, 42, (2009)

7. C. Bouby, D. Fortuné, C. Vallée, W. Pietraszkiewicz, Zeitschrift fur Angewandte Mathematik und Mechanik, 85, (2005)

8. T., Monnet, M., Begon, C., Vallée, P., Lacouture, Biomechanics: Principles, Trends and Applications (2010)

9. Sati, M., de Guise, J.A., Larouche, S., Drouin, G. The Knee, 3, (1996)

10. J. Fuller, L.J. Liu, M.C., Murphy, R.W., Mann, R.W., Human Movement Science, 16, (1997)

11. G. Wu, P.R. Cavanagh, P.R., Journal of Biomechanics, 28, (1995)

12. A.Cappozzo, F., Catani, U., Della Croce, A., Leardini, A. Clinical Biomechanichs, 10 (1995)

13. J.H. Challis, M.T.G., Pain,Exercise and Sport Sciences Reviews, 36,(2008) 